\title{
Application of the Hough transform to correct for linear variation of background illumination in images
}

\author{
M. NIXON \\ Department of Electronics and Information Engineering, University of Southampton, Highfield, Southampton, S09 $5 \mathrm{NH}$, \\ United Kingdom
}

Received 24 September 1984

Revised 23 December 1984

\begin{abstract}
The Hough transform has been formulated to detect areas of linear brightness variation within an image. The formulation leads to significant computational advantage involving a restricted parameter search space. Two applications are included to demonstrate the efficacy of this technique.
\end{abstract}

Key words: Picture processing, pattern recognition. Hough transform, shading correction.

\section{Introduction}

The classical Hough transform (1962) is a fast point-pattern matching technique used mainly to determine whether an analytically defined shape may be detected within an image. Common application of this technique uses feature extraction, e.g. edge detection, to form an image from which an analytical shape may be determined.

For a feature $F$ governed by a set of parameters $f_{i}$ the Hough technique involves search of an image for all points which match the analytical description of the feature for all $f_{i}$ within practical limits. The maximal count of matching point pairs for a particular set of parameters may then be adjudged to appertain to that feature under consideration.

For example, to detect a circle one may search for matching point pairs in a gradient image which satisfy the relation

$$
\left(y-y_{0}\right)^{2}+\left(x-x_{0}\right)^{2}=r^{2}
$$

for all reasonable values of the coordinates of the centre, $x_{0}$ and $y_{0}$, and the radius $r$. The maximal count for a particular $x_{0}, y_{0}$, and $r$ may be adjudged to be a detected circle described by those parameters.

Use of the Hough transform to detect circles in images has been reported (Kimme et al., 1975) and has been applied successfully within this department to locate glasses and determine their type (Bradley, 1984).

Extensions to the Hough transform technique include the generalised Hough transform (Ballard, 1981) which may be used to detect arbitrarily specific shapes, rather than analytically defined shapes, and which finds application in shape recognition etc.

This paper presents the use of the Hough transform technique to detect areas of linear brightness variation within an image. Such brightness variations occur naturally within images either due to reflectance properties, or in background illumination caused by lighting conditions or other effects. 


\section{Application to areas of known brightness variation}

A linear brightness distribution may be characterised by

$$
f(x, y)=m_{x} x+m_{y} y+c .
$$

Application of the classical Hough technique involves definition of an appropriate parameter space for $m_{x}, m_{y}$ and $c$. An accumulator array then contains a count of those points matching the variation defined by a particular $m_{x}, m_{y}$ and $c$. Following search through an image for each set within the defined parameter space, array maxima correspond to sets of collinear points and the maximum in the array may be selected as the most satisfactory brightness variation.

Reformulation of this technique to detect matching points pairs affords significant computational advantage. This advantage is acquired by effective removal of the requirement to search an image for all values of the parameter $c$ in eq. 1. The range of values for this parameter may be extensive which would therefore incur much computational effort. The technique requires search through an image for those point pairs which satisfy the relation

$$
p(i, j)=p\left(i-c_{i}, j-c_{j}\right)+c_{m} .
$$

The derivation of this relation is presented in the Appendix. In this relation $c_{i}$ and $c_{j}$ are effectively the reciprocal of the brightness gradients along the $i$ and $j$ axes respectively $\left(m_{x}\right.$ and $\left.m_{y}\right), c_{m}$ is an off-

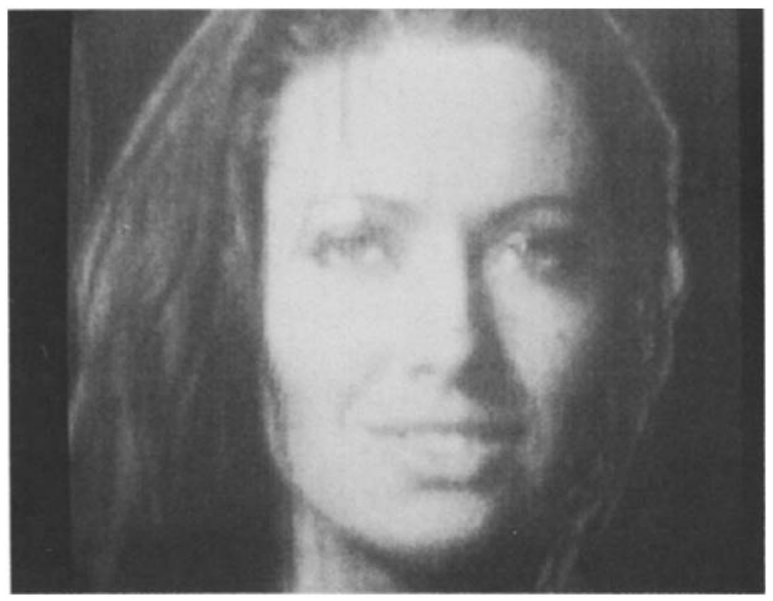

Figure 1. Original image. set $\left(c_{m}=1\right.$ if $m_{x}=0$ or $m_{y}=0, c_{m}=2$ if $m_{x} \neq 0$ and $m_{y} \neq 0$ ).

\section{Implementation}

This technique has been implemented using the SAIDIE image processing development system within the Department of Electronics and Information Engineering at the University of Southampton. This system comprises a framestore of $128 \times 128$ pixels of 256 gray levels, a vidicon CCTV camera and a PDP LSI-11/23 microcomputer plus ancillary devices. Programs for this system are written in FORTRAN IV and run under the RT-11 operating system. Photographs presented in this report were taken from a TV monitor picture with unchanged brightness and contrast levels using an oscilloscope camera of fixed focal length and aperture.

\section{Results}

Figure 1 shows an original image to which is added an image of linear brightness variation, Figure 2, to form a corrupted image Figure 3 . Figure 2 was produced using brightness gradients $c_{i}, c_{j}=0,1$. The original offset ( $c$ in eq. 1) to the brightness variation of Figure 2 was zero (black). Application of the Hough technique involved search through $c_{i} \in 0,10, c_{j} \in 0,10$ and $c_{m} \in 1,2$; the maximal count was found for $c_{i}, c_{j}, c_{m}=0,1,1$,

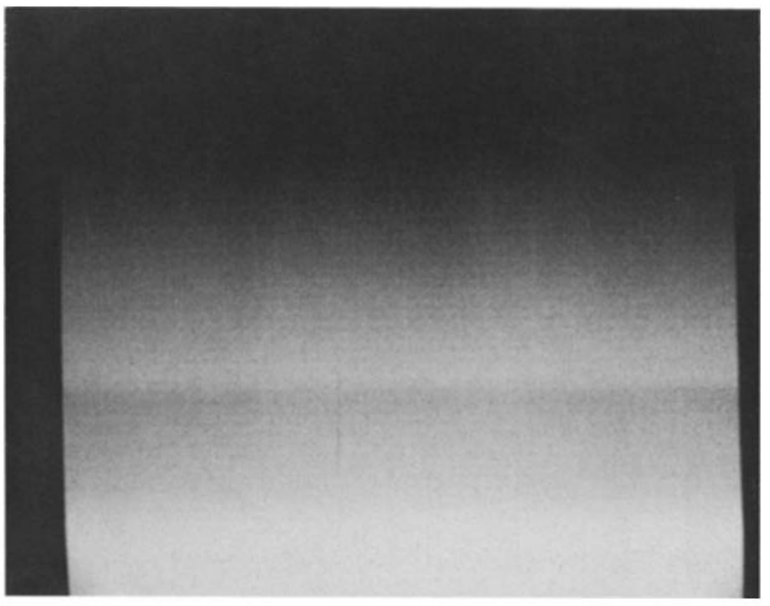

Figure 2. Linear brightness variation. 


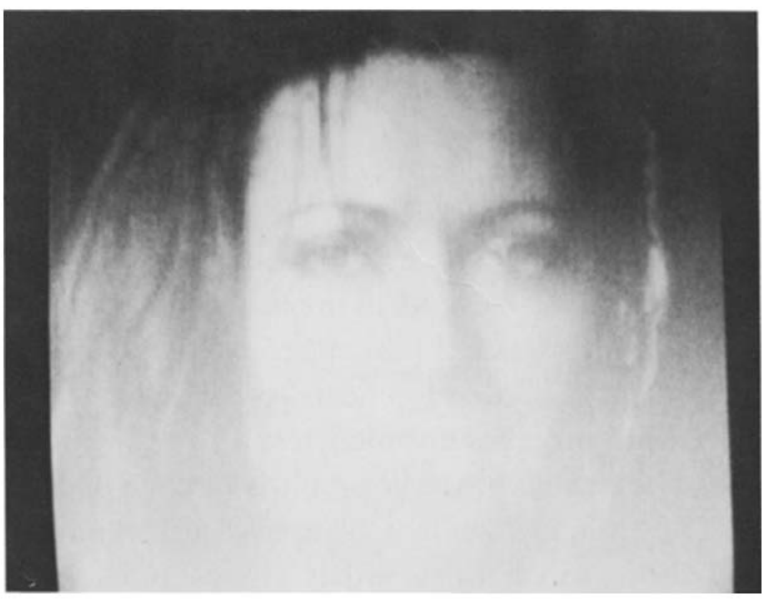

Figure 3. Corrupted image.

those parameters by which the variation of Figure 2 was defined. Subtraction of this brightness variation from Figure 3 led to a 'restored' image Figure 4 which when compared with Figure 3 and Figure 1 illustrates that such restoration has resulted in a marked increase in picture quality.

Figure 5 presents an image of die on a black background though with poor lighting conditions. The die was illuminated using a single flood lamp but this lamp was partially obscured resulting in the observed brightness variation across the background. Application of the Hough technique resulted in a best fit for $c_{i}, c_{j}, c_{m}=0,3,1$. These values imply a brightness variation along the vertical axis only which may be corroborated by visual inspection of Figure 5 . The variation described by these parameters was then subtracted from the image of Figure 5 to form the image of the image of Figure 6, from which it may be observed that com-

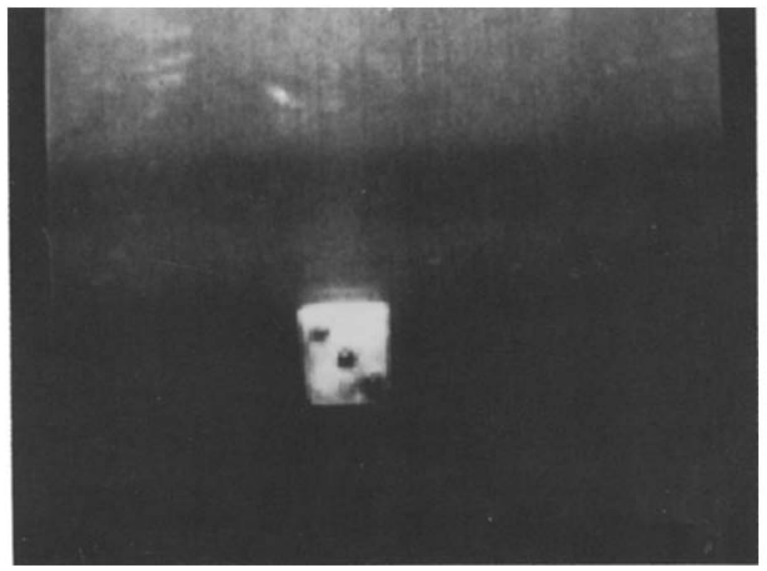

Figure 5 . Original image of a die.

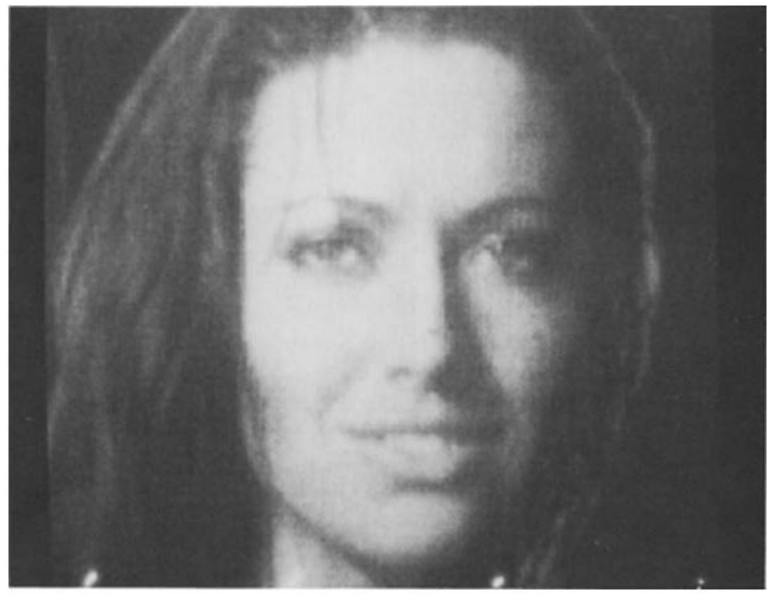

Figure 4 . Resulting image.

pensation for poor lighting has led to an image of improved quality. It should be noted however that the brightness variation determined using this technique was merely that variation for which most points in the original image matched a particular brightness variation. It may prove beneficial to extend the technique to employ a criterion of minimum least-squared error to achieve an optimal fit.

It also should be noted that the image of Figure 5 was illuminated using a single source only. Automated restoration using this technique in the presence of multiple light sources or where an object possesses surfaces with differing reflective properties may require study of lighting geometry and incorporation of segmentation according to brightness distribution within areas of an image.

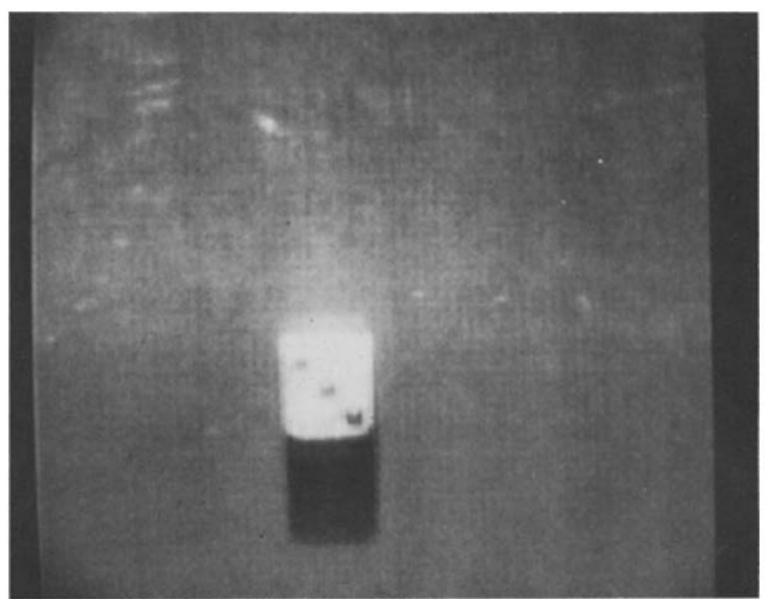

Figure 6. Resulting image. 


\section{Conclusions}

The Hough transform technique has been reformulated to determine areas of linear brightness variation within an image. Determination of these areas makes feasible their suppression thereby restoring an image. Application of this technique has resulted in images of greatly improved quality, and has significant advantage in terms of speed and precision.

The technique is at present limited to linear brightness variations across an image but further extension may make feasible shape extraction, perspective analysis and the enhancement to compensate for poor lighting.

\section{Appendix. Derivation of equation 2}

Consider two points within an image of size $N_{i} \times N_{j}$ pixels, $p\left(i_{1}, j_{1}\right)$ and $p\left(i_{2}, j_{2}\right)\left(i_{1}, i_{2} \in 1, N_{i}\right.$, $\left.\mathrm{j}_{1}, j_{2} \in 1, N_{j}\right)$, which are both of the same linear brightness variation which exists along both axes. These points may be described by

$$
\begin{aligned}
& p\left(i_{1}, j_{1}\right)=m_{x} i_{1}+m_{y} j_{1}+c, \\
& p\left(i_{2}, j_{2}\right)=m_{x} i_{2}+m_{y} j_{2}+c .
\end{aligned}
$$

Then

$$
p\left(i_{1}, j_{1}\right)-p\left(i_{2}, j_{2}\right)=m_{x}\left(i_{1}-i_{2}\right)+m_{y}\left(j_{1}-j_{2}\right) .
$$

Substituting $i_{2}=i_{1}-c_{i}$ and $j_{2}=j_{1}-c_{j}$, it follows

$$
p\left(i_{1}, j_{1}\right)-p\left(i_{1}-c_{l}, j_{1}-c_{j}\right)=m_{x} c_{i}+m_{y} c_{j} .
$$

For $c_{i}=1 / m_{x}$ and $c_{j}=1 / m_{y}$, the algorithm to detect a shading variation along both axes is given by

$$
p\left(i_{1}, j_{1}\right)=p\left(i_{1}-c_{i}, j_{1}-c_{j}\right)+2 .
$$

It is then possible to search for matching points involving a parameter space of two parameters only, not involving the parameter $c$.

Special cases exist when the brightness variation is along a single axis (i.e. either $m_{x}$ or $m_{y}=0$ ). In these cases redevelopment of the detection algorithm provides a similar result to eq. 3 , but in which the value of the constant is unity. The complete formula which may be used to detect a linear variation, which exists either along both axes or along a single axis, is given by

$$
p(i, j)=p\left(i-c_{i}, j-c_{j}\right)+c_{m},
$$

with

$$
\begin{cases}c_{m}=2, & m_{x}, m_{y} \neq 0, \\ c_{m}=1, c_{i}=0, & m_{x}=0, m_{y} \neq 0, \\ c_{m}=1, c_{j}=0, & m_{x} \neq 0, m_{y}=0 .\end{cases}
$$

\section{References}

Ballard, D.H. (1981). Generalising the Hough transform to detect arbitrary shapes. Pattern Recognition 13 (2), 111-122.

Bradley, S.J. (1984). Glass location and recognition via image processing. Report, Department of Electronics and Information Engineering, University of Southampton.

Hough, P.V.C. (1962). Method and Mean for Recognising Complex Patterns, U.S. Patent 3069654.

Kimme, C., D.H. Ballard and J. Sklansky, (1973). Finding circles by an array of accumulators. Comm. Ass. Comput. Mach. 1, 120-122. 